\title{
Application of Optimal Ellipsoid Fitting for Curvature Change Tracking in Main Reflector of Complex Antenna Structures
}

\author{
Hyukgil Kim ${ }^{1}$ and Sungnam Hong ${ }^{2 *}$ \\ ${ }^{1}$ MAC Development Team, Hyundai MnSOFT, \\ Hyundai Motor Building, Wonhyoro 74, Seoul 04365, Republic of Korea \\ ${ }^{2}$ Department of Ocean Civil Engineering, Gyeongsang National University, \\ Engineering Building, Cheondaegukchi 38, Tongyeong 53064, Republic of Korea
}

(Received May 20, 2019; accepted September 27, 2019)

Keywords: VLBI, main reflector, curvature, 3D ellipsoid fitting, close-range photogrammetry

In this study, a curvature change tracking program was developed to evaluate curvature changes in the main reflector of a very long baseline interferometry (VLBI) antenna structure. The data from the geometric model that was developed by fitting the main reflector into a threedimensional (3D) ellipsoid model were used as the fundamental data for the tracking program. The 3D ellipsoid fitting model was established using the 3D position coordinates of the feature points on the reflector surface extracted through nontarget-based close-range photogrammetry. To enhance the fitting accuracy of the 3D ellipsoid model, the optimal parameters were calculated by minimizing the residuals between the conjugate points and the ellipsoid fitting model and by removing outliers. As a result, it was statistically confirmed that the fitting accuracy of the ellipsoid model was improved. The improved ellipsoid model was incorporated into the curvature change tracking program. The developed program will be used to evaluate the structural stability of the main reflector based on periodic curvature calculations, which can also be used as fundamental data for repair and reinforcement work in the future.

\section{Introduction}

Very long baseline interferometry (VLBI) is a technology for precisely determining the location of a measurement point by analyzing the differences in signal arrival time between observation stations. A VLBI antenna mostly consists of a parabolic antenna constructed with a diameter range from 20 to $30 \mathrm{~m}$ and has a reflector surface, which comprises several hundred metal panels. The integrity of the shape of the parabolic reflector surface is critical to the antenna's gain performance. Deviation from the design shape can introduce measurement biases, which influence VLBI observations. This is because changes in the focal length of the main reflector or local deformations on the reflector's surface directly result in biased signal paths and, thus, biased baseline estimates. ${ }^{(1,2)}$

To accurately determine changes in reflector shape, points on the structure at which deformations are likely to occur should be selected and periodically monitored. An "Corresponding author: e-mail: snhong@gnu.ac.kr https://oi.org/10.18494/SAM.2019.2448 
accurate evaluation of the deformation sustained by VLBI antenna structures after extreme environmental events such as typhoons or earthquakes is critical to the determination of the safety and suitability of structures for their future occupancy. A quantitative measurement of the deformation is an effective means of providing a basis for a more reliable estimation of VLBI antenna structure safety. In a VLBI antenna structure, the curvature variations and patterns on the inner surface of the main reflector are the most important indicators used to estimate durability. To measure changes in curvature and prevent resultant damage, it is necessary to establish techniques for tracking curvature changes.

Most available approaches in this area involve the application of linear variable differential transformers (LVDTs), demountable mechanical strain gauges (DEMECs), extensometers, and fiber optics. However, a general disadvantage of these techniques is their pointwise and onedimensional (1D) measurement capability. If simultaneous two- or three-dimensional (2D or 3D) measurements at several locations are required, the instrumental workload becomes rather large. The techniques are generally not suited for tasks requiring a large number of measurement points distributed over an object surface or for complete surface measurements. ${ }^{(3)}$

In these cases, close-range photogrammetry is a valuable alternative for the design of powerful and flexible measurement tools. The application of close-range photogrammetry will generally allow the simultaneous measurement of the deformation or displacement at an almost arbitrary number of locations over the camera's field of view. Data processing can be highly automated and fast, allowing real-time tracking at the camera imaging rate. ${ }^{(3)}$ The potential accuracy of close-range photogrammetry is generally at the sub-millimeter level in the case of measurement of the reflector target and at the millimeter level in the case of measurement of the nontarget.

Fitting algebraic or geometric surfaces to 3D data is a pervasive problem in numerous fields of science and engineering. Primitive 3D fitting models involve fitting a quadratic surface to a set of points in space, which has applications in 3D reconstruction, pose estimation, the restricted stereo correspondence problem, and object recognition. ${ }^{(4-10)}$ One of the most used fitting models is the ellipsoid, which is of paramount importance in several fields.

There have been numerous approaches toward solving the ellipsoid fitting problem. Previous work can be classified into two categories, namely, minimizing the algebraic distance and minimizing the geometric distance, according to their error distance definitions. ${ }^{(11-19)}$ The problems with geometric fitting (i.e., ineffective initialization, nonuniform point sampling, and heavy noise) are likely to result in an ineffective local minimum solution. Furthermore, it is challenging to deduce the geometric error in high-dimensional spaces. In contrast, algebraic fitting is significantly more straightforward owing to its convexity. Thus, it has been greatly preferred in the literature owing to its more convenient numerical treatment and more effective statistical interpretation. ${ }^{(20)}$

In this study, we propose a new curvature change tracking measurement technique based on a 3D geometrical model and close-range photogrammetry. An optimal geometric model of scattered data in a 3D space was constructed by algebraic ellipsoid fitting, and the data were used as fundamental data for calculating the curvature changes of the main reflector. The algebraic ellipsoid fitting problem can be mapped to an optimization problem that can be solved 
using the least-squares (LS) method. The parameters for the construction of an optimal 3D ellipsoid model for the main reflector were calculated by applying an algorithm that enhances the fitting accuracy while applying 3D ellipsoid fitting using the LS method. Finally, to increase the accuracy of geodetic and astrometrical measurements performed by the VLBI antenna, a program that could periodically calculate the curvature changes of the main reflector with minimum labor and cost was developed.

\section{Study Subject and Experimental Configuration}

To specify the optimal ellipsoid fitting method for detecting the curvature changes in a main reflector, in which multiple feature points are distributed, the main reflector of the VLBI antenna located in Sejong, a city in central South Korea, was selected as the target antenna. The VLBI antenna is of the Cassegrain type, which consists of a main reflector with a diameter of $22 \mathrm{~m}$ and a subreflector with a diameter of $2.2 \mathrm{~m}$. The general specifications for the VLBI antenna structure are presented in Table 1.

\subsection{Nontarget-based photogrammetry}

Close-range photogrammetry is a technique for determining the 3D geometry (location, size, and shape) of physical objects by measuring and analyzing their 2D photographs obtained from the ground; it has been frequently applied to measure the displacement and deformation of facilities. ${ }^{(21-24)}$ The technological advances of close-range photogrammetry have matured to the point that they can now be implemented as measurement tools to obtain a detailed understanding of the mechanical deformations and full-field strain distribution of large scale composite structures. This technique is a noncontact measurement technique that uses images captured simultaneously by a pair of digital cameras to track prescribed targets placed on a structure. Using the principle of close-range photogrammetry, a full-field 3D structural shape consisting of several thousand measurement points can be acquired.

To successfully apply close-range photogrammetry, various types of targets should be bonded to the subjects, and an accurate measurement process for each target should be established. The target configuration can be classified into the utilization of the feature points distinguished from the other pixels in the captured image and the attachment of the separate

Table 1

Specifications of VLBI antenna structure used in the study.

\begin{tabular}{lc}
\hline Classifications & Values \\
\hline Antenna type & Cassegrain \\
Main reflector diameter & $22 \mathrm{~m}$ \\
Subreflector diameter & $2.2 \mathrm{~m}$ \\
Receiving frequency band & $2,8,22$, and $43 \mathrm{GHz}$ \\
Antenna illumination efficiency & $-60 \%$ \\
Precision of antenna pointing & $0.0131^{\circ}(\mathrm{RMS})$ \\
Precision of main reflector curved surface & 86 \\
Driving range & Azimuth: $\pm 270^{\circ}$, elevation: $0-90^{\circ}$ \\
Driving speed & Azimuth and elevation $5^{\circ} / \mathrm{s}$ \\
\hline
\end{tabular}


targets. If the feature points are used as targets, nontarget-based close-range photogrammetry is advantageous for implementing a noncontact method that does not attach any targets to structures.

An initialization process should be performed to calculate the interior and exterior orientation parameters of the cameras before performing close-range photogrammetry for each target using nonmetric cameras. The precise values for the interior and exterior orientation parameters are calculated from the actual coordinates of the feature points measured by a total station survey. The actual coordinates for the targets are used only once in the calculation of the camera calibration and orientation parameters. However, the orientation parameters of the camera determined through the actual coordinates are continuously used until the camera is replaced. Therefore, the actual coordinate measurement of the target and the camera calibration task should be conducted very accurately to determine the precise orientation parameters through the initialization process.

In this study, close-range photogrammetry based on direct linear transformation (DLT) and a self-calibration bundle adjustment with additional parameters are used to obtain the 3D position coordinates of the feature points. ${ }^{(25,26)}$ DLT and bundle adjustment are precise camera calibration techniques and can be used as algorithms for constructing a fast and automated camera calibration process without using specific commercial software packages.

The 3D position coordinates of the feature points were calculated with mm-level positioning accuracy by applying nontarget-based close-range photogrammetry to the main reflector of the VLBI antenna, wherein multiple feature points are distributed on the surface of the structure. High-resolution IP cameras were utilized as measurement sensors for the application of closerange photogrammetry. To solve the problems of IP cameras with a lower geometric stability than metric cameras, quantitative figures were obtained to apply consumer-grade digital cameras to close-range photogrammetry, through the precise calculation of the interior and exterior orientation parameters.

The procedure for determining the 3D position coordinates mentioned above was first applied in a study conducted by Kim et al. ${ }^{(25)}$ The close-range photogrammetry software developed in the previous study was used to determine the 3D position coordinates of the feature points in this study.

\subsection{Experimental configuration}

Figure 1 shows the experimental configuration for applying close-range photogrammetry to the main reflector of the VLBI antenna at the Space Geodetic Observation Center (SGOC), which is operated by the National Geographic Information Institute in South Korea. To perform the ground control point survey for feature points uniformly distributed on the inner surface of the main reflector, a total station was installed in the fixed pillar closest to the VLBI position, and a local coordinate system was set. The total station used to conduct the ground control point survey was an S8 Total Station from Trimble. The VLBI antenna was pointed toward the azimuth, and the elevation angles were selected such that its front faced the total station installation direction at fixed intervals. 


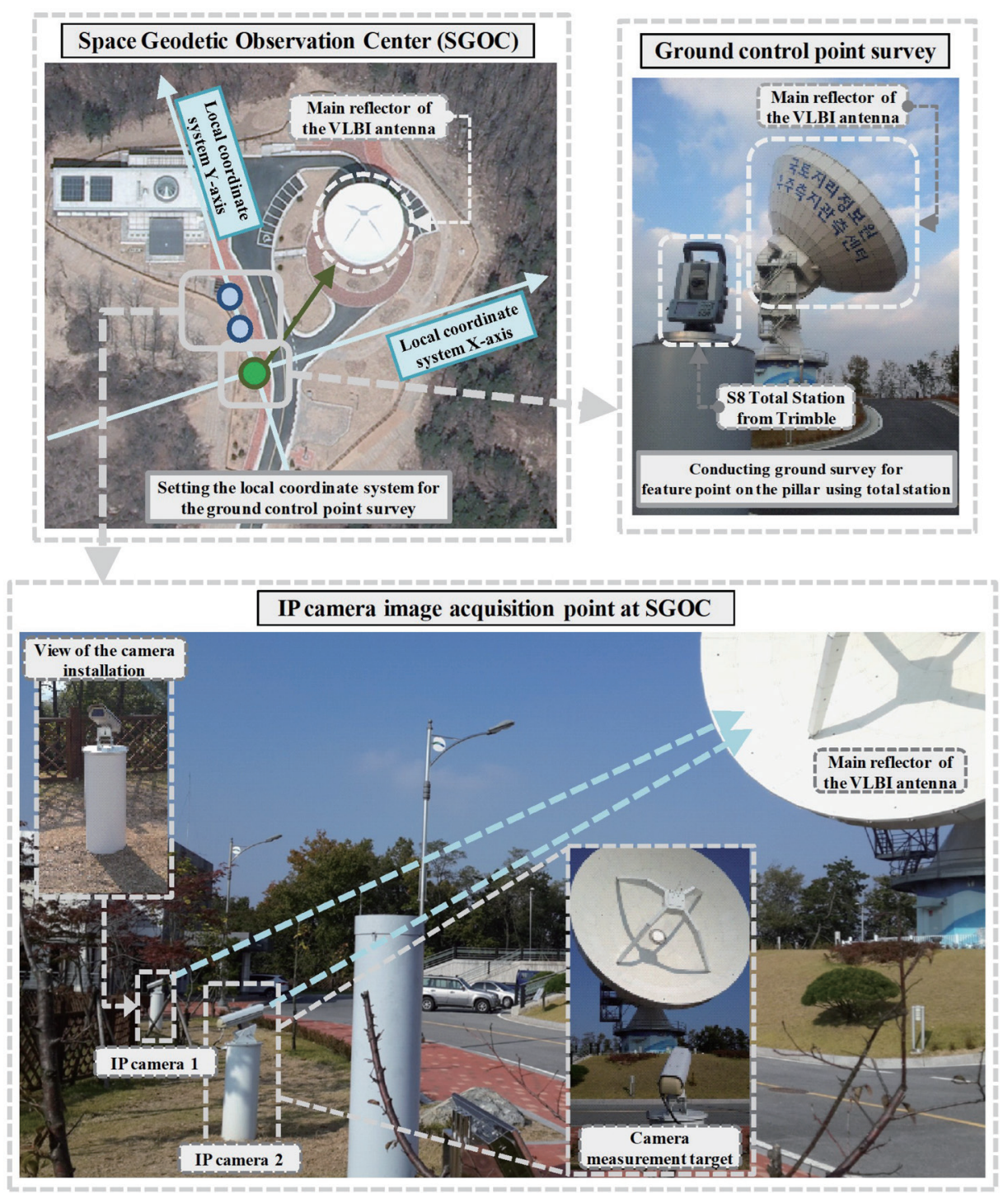

Fig. 1. (Color online) Experimental configuration for close-range photogrammetry application to main reflector of VLBI antenna at the SGOC. ${ }^{(25)}$

Attaching a reflective target on the inner surface of the main reflector for a ground control point survey is likely to reduce the positioning accuracy of the VLBI antenna owing to radio interference. Thus, the feature points were measured by nontarget total station measurements. The measurements using the total station exhibited accuracy and error ranges of $\pm(2 \mathrm{~mm}+$ $2 \mathrm{ppm})$ in the standard mode and $\pm(4 \mathrm{~mm}+2 \mathrm{ppm})$ in the tracking mode.

For close-range photogrammetry, stereo images of the main reflector were captured by two IP cameras, as shown in Fig. 1. Arecont Vision AV10005DN cameras, which can measure 10-megapixel images at $6 \mathrm{fps}$, were used. As the cameras are used as references for the deformation measurement, they must be installed firmly so that their positions and pointing 
directions do not change. The fixed focal lengths of charge-coupled device (CCD) cameras 1 and 2 in Fig. 2 were 12.466 and $10.367 \mathrm{~mm}$, respectively. The distances from these two cameras to the VLBI antenna were approximately 37 and $41 \mathrm{~m}$, respectively. The image size of the cameras was $2560(\mathrm{H}) \times 1920(\mathrm{~V})$ pixels, the sensor size was $1 / 2.3$ ", and the pixel pitch was $1.67 \mu \mathrm{m}$.

Figure 2 shows the 3D position coordinates of the feature points on the inner surface of the VLBI antenna, calculated using the close-range photogrammetry software developed by Kim et al. ${ }^{(25)}$ Using their software, we calculated four residuals for the 3D position coordinates determined by LS estimation. The precision of the results was verified using the standard deviation of the residuals, as shown in Fig. 2. The mean precision of the 3D position coordinates was $2 \mathrm{~mm}$, which was regarded as sufficient for detecting the excessive deformation of the VLBI antenna surface.

\section{Study of Method for Optimal Ellipsoid Fitting}

\subsection{Geometric modeling of main reflector of VLBI antenna}

The development of a technique for fitting a specific ellipsoid to a set of points distributed within a 3D space is a thoroughly studied problem, with numerous applications in the fields of visual pattern recognition, astronomy, digital image analysis, computer vision, and computer graphics. ${ }^{(27-29)}$ Using an ellipsoid fitting technique, we fitted the 3D position coordinates of the conjugate points on the main reflector into a 3D ellipsoid equation and constructed a geometric model of the main reflector. The data from this geometric model were used as fundamental data for calculating the curvature changes of the main reflector.

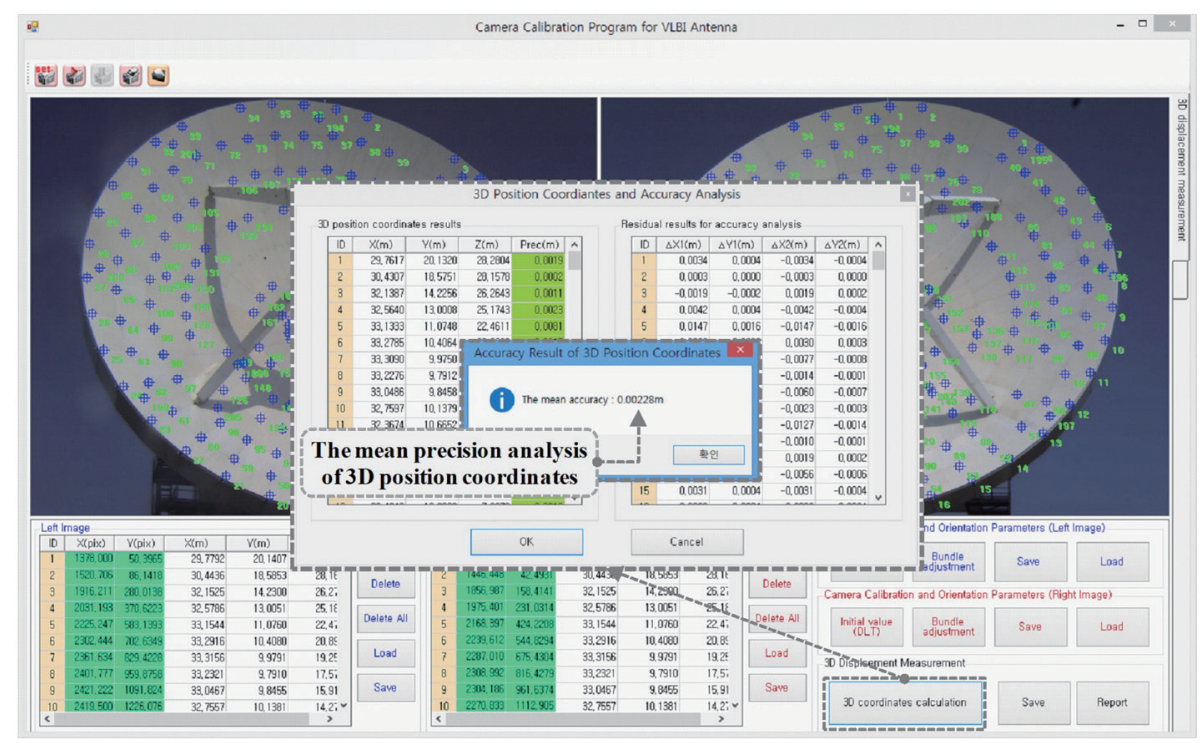

Fig. 2. (Color online) Measurement and accuracy analysis results for 3D position coordinates for conjugate points calculated using close-range photogrammetry software. ${ }^{(25)}$ 
An ellipsoid equation for a 3D space can be expressed as a second-order algebraic equation as $^{(29)}$

$$
a x^{2}+b y^{2}+c z^{2}+2 f y z+2 g x z+2 h x y+2 p x+2 q y+2 r z+d=0
$$

To construct a fitting model of a main reflector, fitting was attempted using the model with the 3D position coordinates of the conjugate points calculated through nontarget-based closerange photogrammetry. However, it was difficult to achieve the fitting accuracy for the required shape modeling of the main reflector. The elliptic, parabolic, and hyperbolic equations can implement 3D shapes by determining the 2D shape of the plane curve of the main reflector and then rotating it around the principal axis. However, the objects used in the fitting in this study were 188 conjugate points in 3D space. To implement an approximate fitting model, a method for enhancing the fitting accuracy by considering an algebraic equation for the 3D shape and eliminating the outliers between the conjugate points and the fitting equation was considered appropriate.

A fitting model for the main reflector was established to calculate the curvature changes of the main reflector of the VLBI antenna. To calculate the Gaussian and mean curvatures of the main reflector, the $(X, Y, Z)$ coordinates of the surface of the 3D fitting model of the main reflector were used. The $(X, Y, Z)$ coordinates used were $2 \mathrm{D}$ arrays of the points on the surface of the fitting model. The determination of the curvature of each $(X, Y, Z)$ coordinate on the surface of the 3D fitting model was conjectured to be capable of efficiently analyzing the curvature distribution for the main reflector. Therefore, to construct a fitting model for the numerous conjugate points distributed in 3D space, a 3D ellipsoid model was constructed using Eq. (1). However, the ten parameters of Eq. (1) together cannot yield an accurate fitting model owing to the high level of uncertainty in the orientation of the principal axis of the quadric. Therefore, the optimal fitting model for the distribution of the conjugate points was determined to be the following 3D ellipsoid equation with the following seven parameters:

$$
a x^{2}+b y^{2}+c z^{2}+2 p x+2 q y+2 r z+d=0 .
$$

The algorithm for fitting the 3D position coordinates of the conjugate points into the algebraically expressed ellipsoid equation in Eq. (2) is presented in Fig. 3. By following the process shown in Fig. 3, the conjugate points distributed within 3D space can be fitted into an ellipsoid equation optimized through the LS method. As a result, the coordinates of the center of the ellipsoid and the radius and direction matrix of the ellipsoid model can be determined, and the values of the seven parameters in the algebraic ellipsoid equation in Eq. (2) can be determined.

An ellipsoid model in 3D space using the center coordinates of the ellipsoid and the radii of the ellipsoid along the three axes is shown in Fig. 4. This figure illustrates the 3D position coordinates of the conjugate points of the stereo images, which are used to express the ellipsoid model, on a 3D graph. 


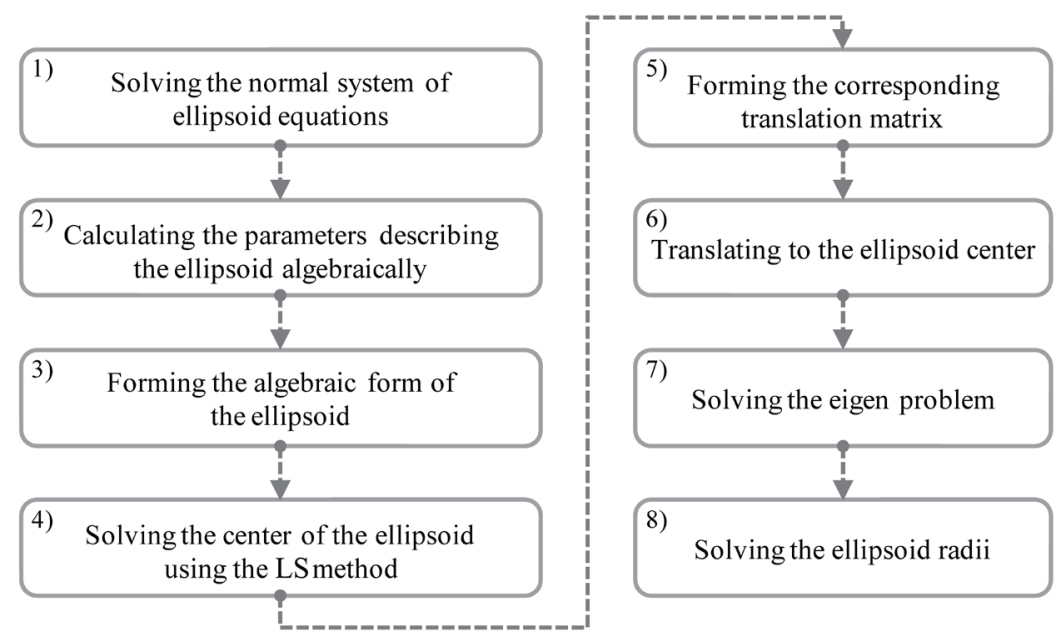

Fig. 3. 3D ellipsoid fitting algorithm for main reflector of VLBI antenna.

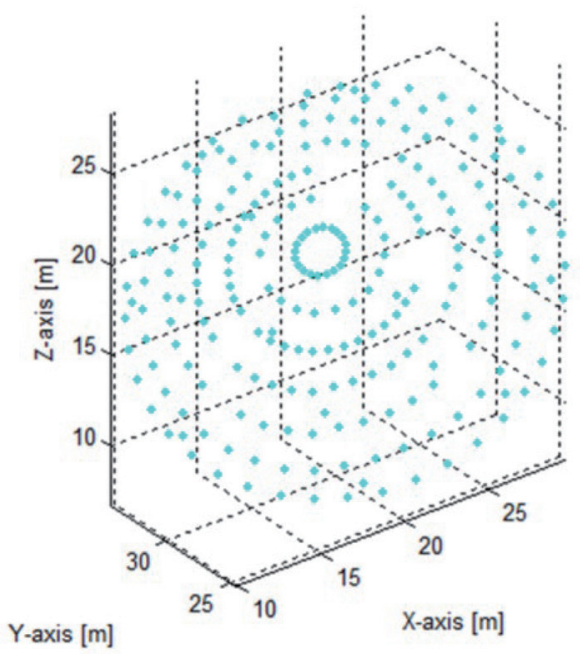

Fig. 4. (Color online) Distribution of 3D position coordinates for conjugate points of stereo image.

Figure 5 shows the results of overlaying the 3D position coordinates of the conjugate points in Fig. 4 on the ellipsoid model constructed using the center coordinates and the radii along the three axes determined through the ellipsoid model fitting process. The 3D position coordinates of the conjugate points were fitted into the ellipsoid model in the appropriate direction.

Figure 6 shows the part of the ellipsoid fitting model that is appropriate for tracking changes in curvature. The figure shows only a portion of the ellipsoid model on the 3D graph to optimally express the curvature change tracking for the main reflector.

\subsection{Curvature calculation of main reflector of VLBI antenna}

The Gaussian and mean curvatures were calculated using the 2D coordinates of the points making up the geometrically fitted ellipsoid model. These curvatures were used to track the 


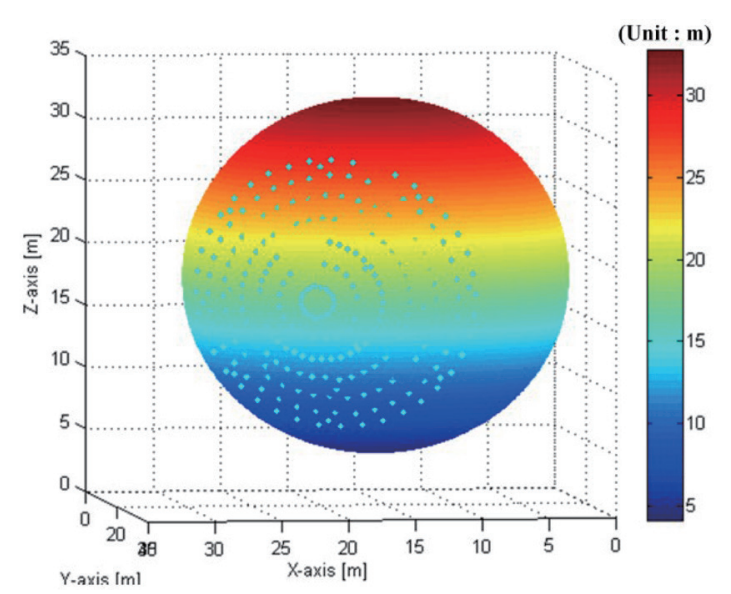

Fig. 5. (Color online) Results of overlaying ellipsoid fitting model and conjugate points.

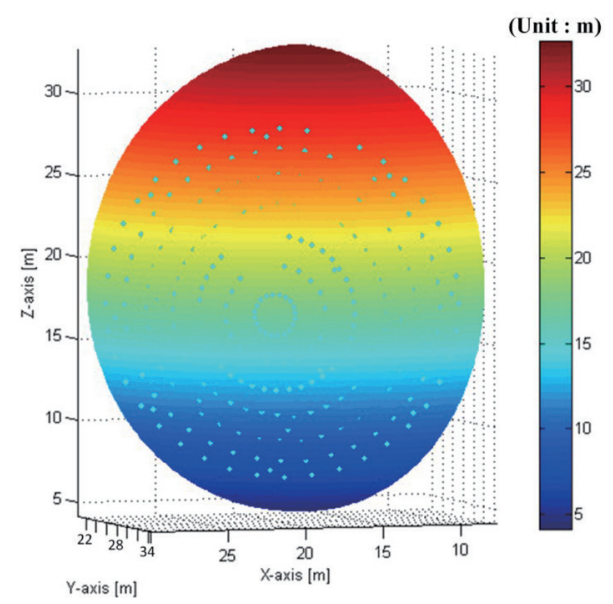

Fig. 6. (Color online) Ellipsoid fitting model of main reflector to optimally express curvature change tracking.
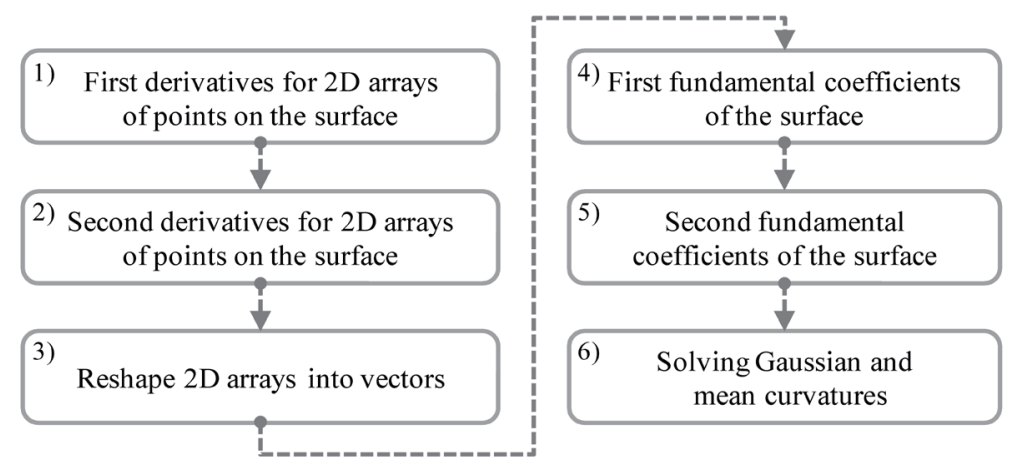

Fig. 7. Gaussian and mean curvature algorithm.

changes in the curvature of the main reflector. Figure 7 illustrates the algorithm for calculating the curvature of the main reflector that was applied as the geometric model in Fig. 6.

Figure 8 shows a 3D graphic representation of the Gaussian and mean curvatures for each point of the ellipsoid fitting model calculated using the algorithm shown in Fig. 7 based on the ellipsoid fitting model for the main reflector shown in Fig. 6. A 3D colored surface was implemented using the curvatures calculated at each position coordinate on the fitting model as a new property value. The fitting model containing the curvatures was used to regularly monitor the deformation of the main reflector by converting the Gaussian and mean curvatures for each point of the ellipsoid fitting model into a database to obtain a time series.

As shown in Fig. 8, the color scaling of the graph was implemented using a range of curvatures for each point of the fitting model, and the curvatures of the fitting model were uniformly distributed throughout the model. The model in Fig. 8 was designed to detect abnormal changes in the curvature of the main reflector by periodically calculating the curvature from the time series within the same section. 


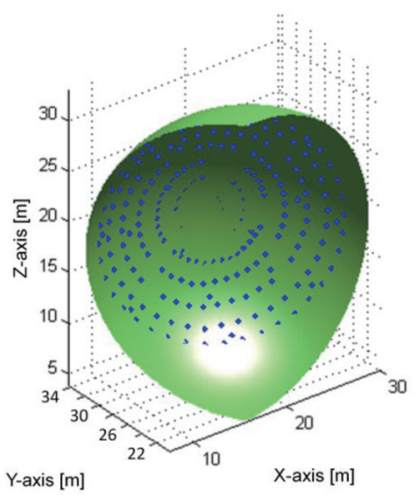

(a)
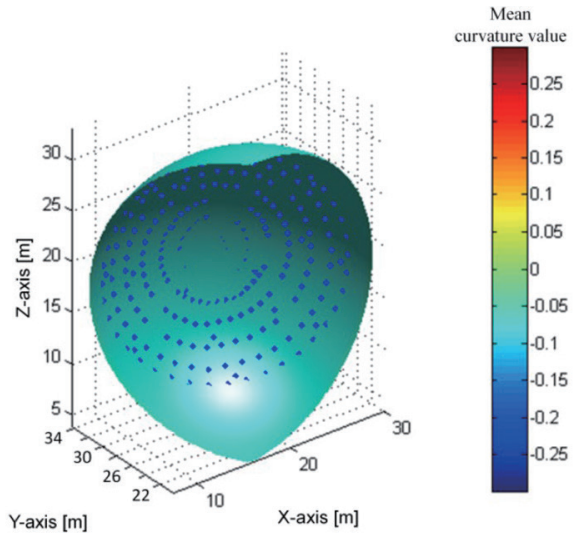

(b)

Fig. 8. (Color online) Ellipsoid fitting model expressed based on distribution of curvatures, (a) distribution of Gaussian curvatures, and (b) distribution of mean curvatures.

\section{Enhancement of Fitting Accuracy for Optimal Modeling}

To enhance the fitting accuracy of the ellipsoid model, the optimal parameters were calculated by minimizing the residuals between the conjugate points and the ellipsoid fitting model and by removing outliers. For this purpose, the residuals between the conjugate points and the ellipsoid fitting model were regarded as distance functions, and the steepest-descent method, which is a nonlinear optimization method, was applied.

To determine the local minimum of a function using a gradient descent, steps proportional to the negative of the gradient of the function at the current point were taken. Using the steepest-descent method, the distance between a point within a space and a random point on the fitted ellipsoid model was calculated as the residual, and the minimum of this distance was determined by iteratively calculating a random point on the ellipsoid that minimizes the distance.

To enhance the fitting accuracy, the standard deviation of all the residuals of the conjugate points was determined. When a residual exceeded the $95 \%$ probable error of the standard deviation, the conjugate point was determined to be an outlier and removed.

The fitting accuracy of the geometric model of the main reflector was enhanced by recalculating the parameters of the ellipsoid fitting model using the conjugate points from which a few of the outliers had been removed and by recalculating the curvatures. The process for improving the fitting accuracy is described in Fig. 9.

The process for determining the nearest point from a point on the ellipsoid fitting model in 3D space is illustrated in Fig. 10. The minimum distance function $\mathrm{J}$ determined using the algorithm in Fig. 10 indicates the minimum distance between the 3D position coordinate of the conjugate point and the ellipsoid fitting model within the 3D space.

The minimum distance function $\mathrm{J}$ was iteratively calculated by the above process, and the minimum $\mathbf{J}$ for each point was defined as the residual of the fitting model. The standard deviation of the set of all residuals, calculated iteratively for each point distributed within 


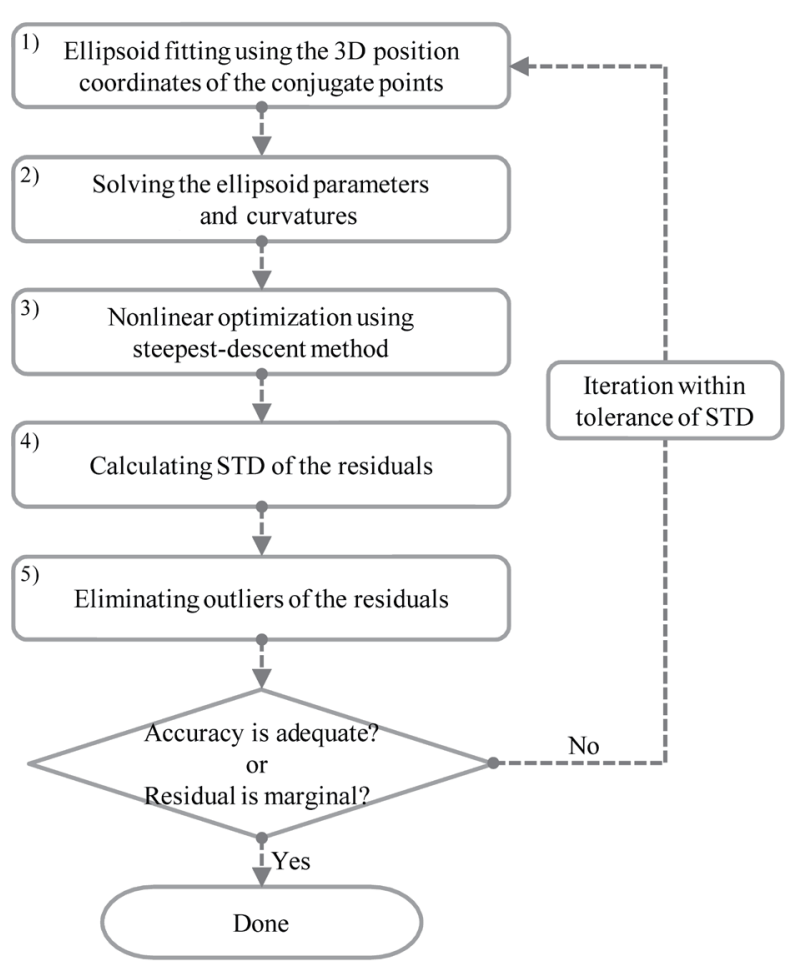

Fig. 9. Process for enhancing fitting accuracy.

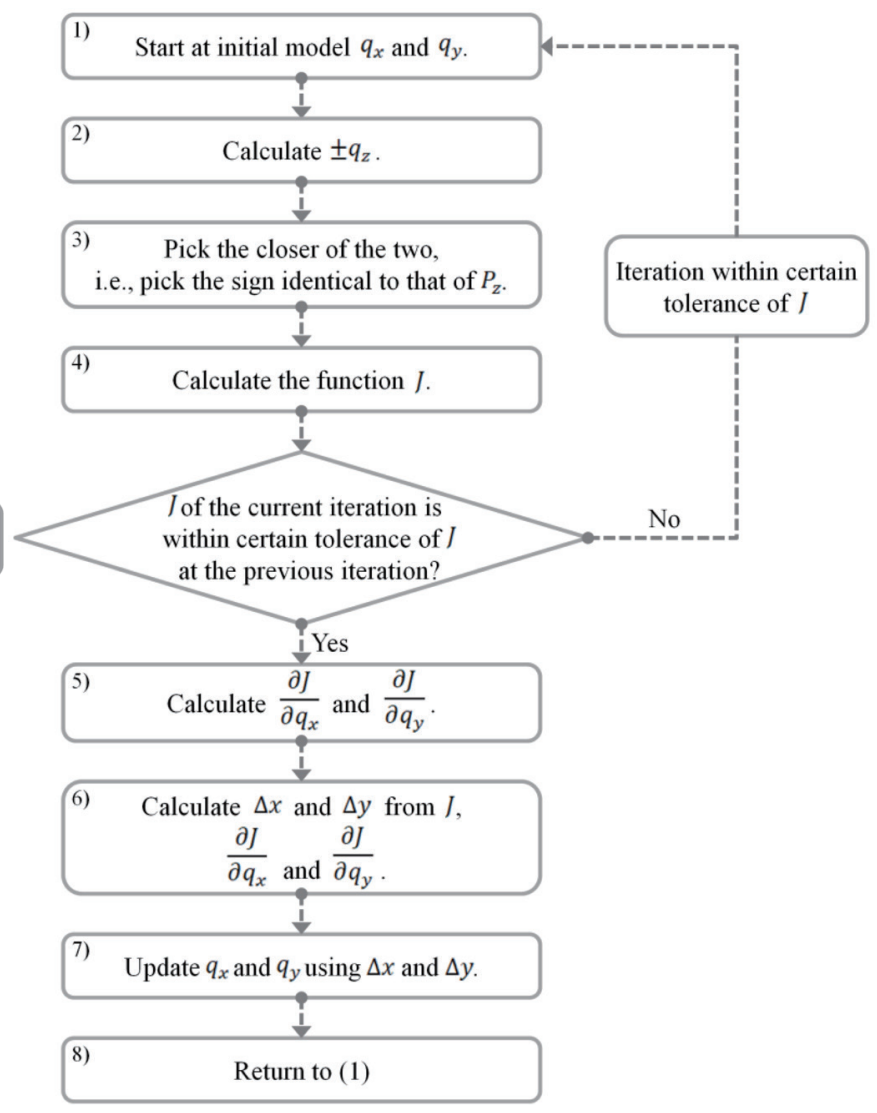

Fig. 10. Algorithm for finding the nearest point from a point on the ellipsoid fitting model.

the 3D space, was determined. The points outside the $95 \%$ probable error of the standard deviation were regarded as outliers and removed. The ellipsoid model fitting was applied again iteratively for the set of points from which outliers had been removed, and the fitting accuracy of the model of the main reflector was enhanced by determining the optimal parameters of the ellipsoid fitting model. The detailed calculation procedure of the algorithm shown in Fig. 10 including equations can be found elsewhere. ${ }^{(26)}$

Figure 11 expresses the residual distribution between the conjugate points and the ellipsoid fitting model. In this figure, the red lines indicate the residuals between the 3D position coordinates of the conjugate points used in the ellipsoid fitting and the fitting model. A longer red line is associated with a larger residual between the conjugate points and the ellipsoid fitting model. Furthermore, the 3D position coordinates of the conjugate points used for fitting the ellipsoid model are represented by the blue dots, and the black circles on the blue dots represent the conjugate points that are the objects of the residual calculation. To enhance the accuracy of the ellipsoid fitting, the conjugate points with large residuals inside the red boxes shown in Fig. 11 were regarded as outliers, and the calculations for removing them were repeatedly conducted. After the first such calculation, the outliers were removed from the red box. Furthermore, the number of points with a relatively long red line decreased throughout the use 


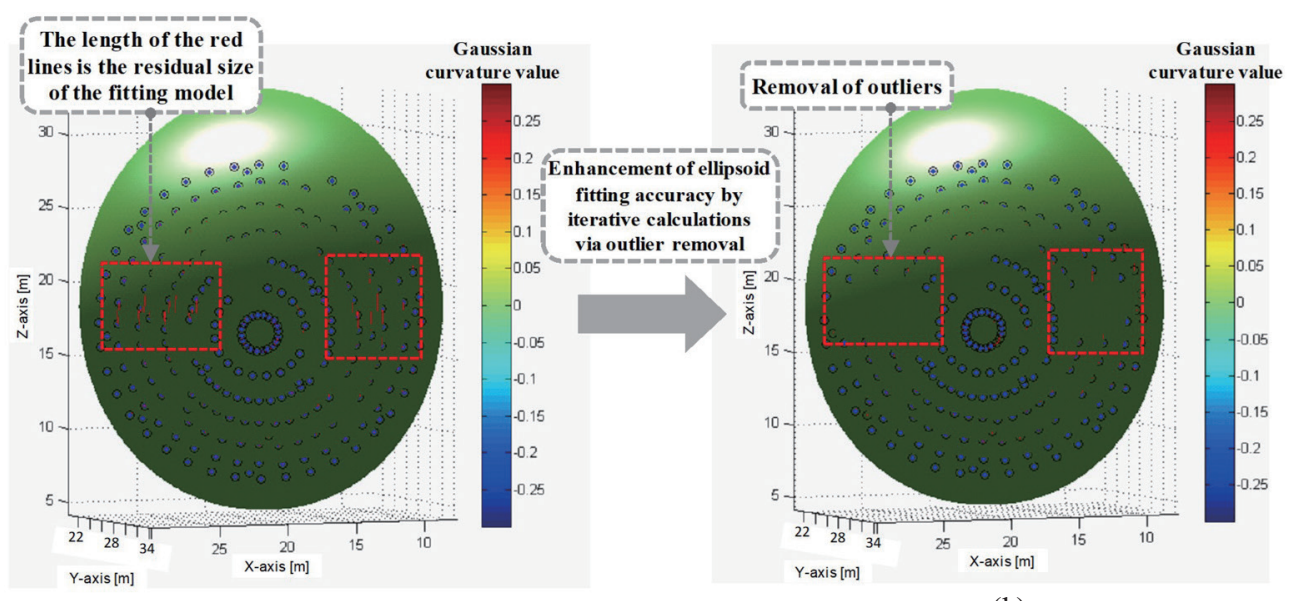

(a)

(b)

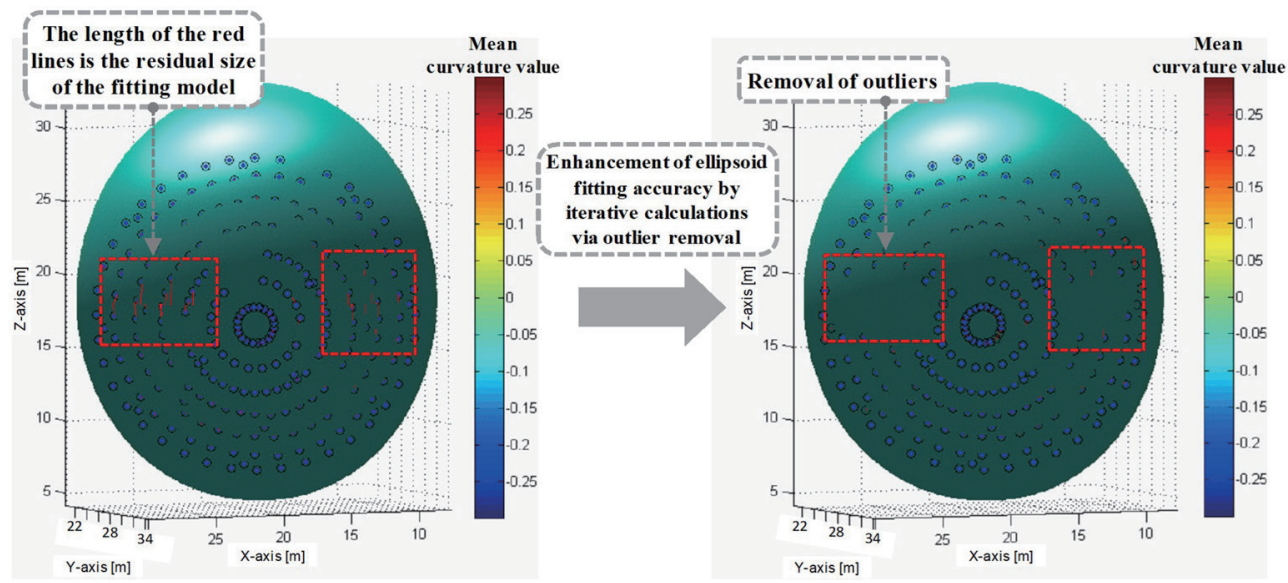

(c)

(d)

Fig. 11. (Color online) Residual distribution between conjugate points and ellipsoid fitting model, (a) initial residual distribution (model expressed by Gaussian curvature), (b) residual distribution obtained by first outlier removal calculation (model expressed by Gaussian curvature), (c) initial residual distribution (model expressed by mean curvature), and (d) residual distribution obtained by first outlier removal calculation (model expressed by mean curvature).

of the fitting model. The fitting accuracy can be enhanced by repeatedly applying ellipsoid fitting to those points whose outliers have been partially removed.

The results of the residual distribution analysis expressed through the red lines revealed that the outer parts of the main reflector fit the ellipsoid model more accurately than the central part. Because the outer parts of the main reflector exhibit a higher likelihood of curvature change, the ellipsoid fitting model can be used for tracking the change in the curvature of the main reflector.

\section{Development of Curvature Change Tracking Program}

A program for calculating the curvature changes of the main reflector was developed, as shown in Fig. 12. MATLAB was used owing to its numerical and GUI features. This program 


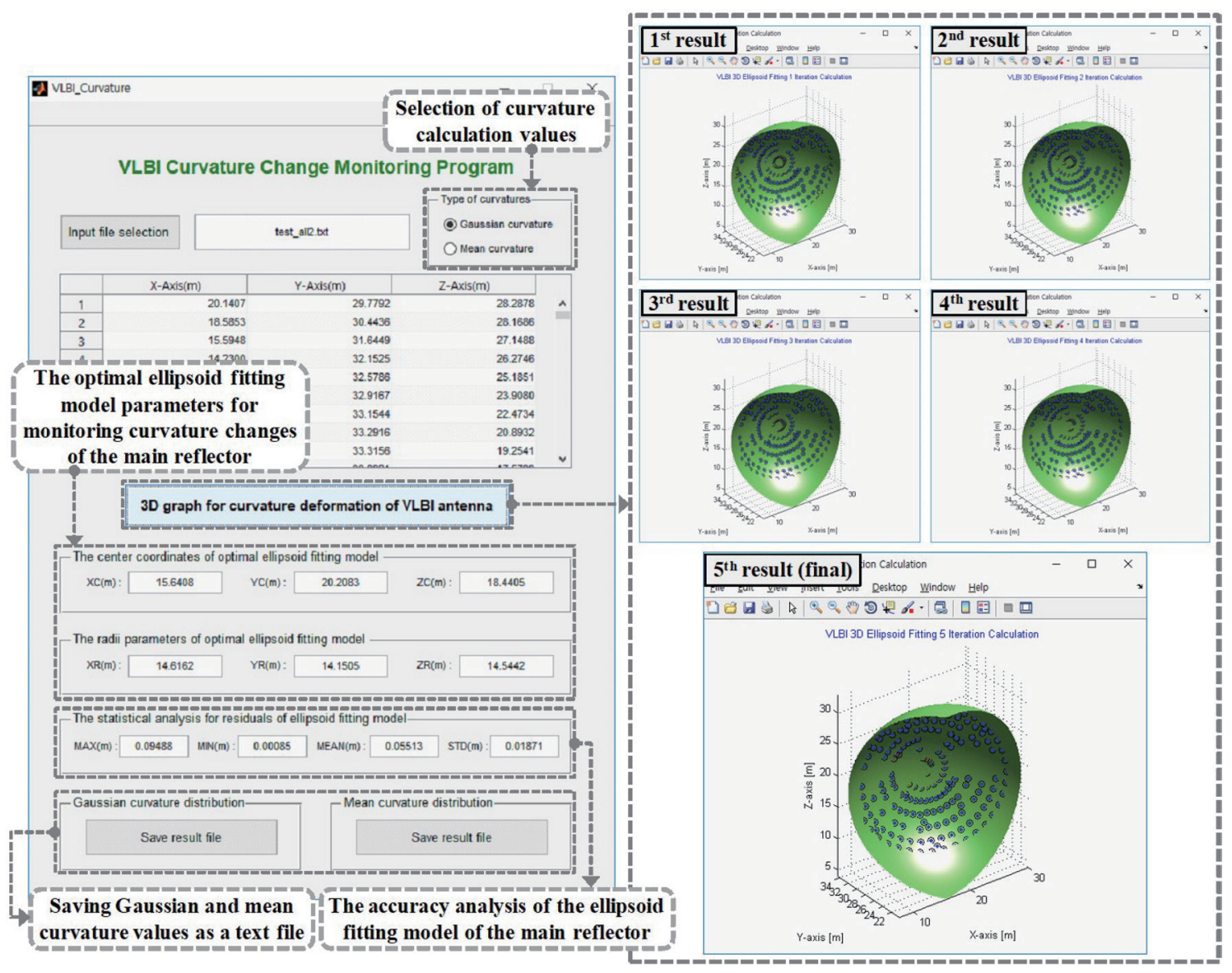

Fig. 12. (Color online) 3D graph results of optimal ellipsoid fitting model achieved through the developed program.

was used to fit the 3D ellipsoid model using the procedure described in Sects. 3 and 4, namely, by deriving the center point and the radius along each axis using the 3D position coordinates of the conjugate points and enhancing the fitting accuracy by iteratively removing the outlier points. Moreover, the Gaussian and mean curvatures were calculated for each point by using the $2 \mathrm{D}$ coordinates of the fitting model.

The program mainly consists of the input part, which loads the 3D position coordinate file for the conjugate points calculated by nontarget-based close-range photogrammetry, and the output part, which displays a 3D graph that has been fitted into the optimal 3D ellipsoid model and model parameters. Furthermore, to calculate the curvature changes of the main reflector through a time-series analysis of the same range, the program was designed to output each curvature result as a text file.

The graphs in Fig. 12 were implemented by expressing the Gaussian curvatures in the model whose ellipsoid fitting process had been completed. The iterative calculations used to determine the optimal parameters of the ellipsoid fitting model were set to stop the calculation process if the standard deviation was $2 \mathrm{~cm}$ or lower or if the amount of data after the outlier removal was $85 \%$ of the initial data or lower. These criteria were determined to prevent loss of the original data owing to excessive data removal and to maintain a fitting accuracy similar to 
Table 2

Results of statistical analysis based on iterative ellipsoid fitting calculations.

\begin{tabular}{lcccc}
\hline Statistical indicator & $\begin{array}{c}\text { Maximum deviation } \\
(\mathrm{m})\end{array}$ & $\begin{array}{c}\text { Minimum deviation } \\
(\mathrm{m})\end{array}$ & $\begin{array}{c}\text { Average deviation } \\
(\mathrm{m})\end{array}$ & $\begin{array}{c}\text { Standard deviation } \\
(\mathrm{m})\end{array}$ \\
\hline First iteration & 0.164 & 0.006 & 0.080 & 0.035 \\
Second iteration & 0.133 & 0.003 & 0.064 & 0.026 \\
Third iteration & 0.114 & 0.002 & 0.058 & 0.021 \\
Fourth iteration & 0.103 & 0.001 & 0.056 & 0.020 \\
Fifth iteration & 0.095 & 0.001 & 0.055 & 0.019 \\
\hline
\end{tabular}

that of the time-series calculations. The curvature changes can be detected from the time-series analysis by identifying the abnormal tendencies.

Table 2 presents the results of the statistical analysis of the residuals based on the iterative ellipsoid fitting calculations through outlier removal. The ellipsoid fitting accuracy was enhanced by applying a statistical confidence interval of 95\% (1.96 $\sigma)$ to the residuals and then identifying and removing the residuals that exceeded this range (outliers). As shown in Table 2, the accuracy of the first iterative calculation was $0.080 \pm 0.035 \mathrm{~m}$, whereas that of the fifth and final iterative calculation improved to $0.055 \pm 0.019 \mathrm{~m}$.

\section{Conclusions}

Deformation measurements are performed periodically to prevent death and injuries due to the damage or collapse of built structures. Deformations and displacements in such structures can occur if they are not constructed on firm ground and are subjected to extreme loading; this causes hazardous shaking owing to crustal movements and external environmental conditions. To maintain the structural integrity under these conditions, it is crucial to make continuous observations using more convenient and cost-effective measurement methods than the traditional methods used to detect deformations and displacements.

For this purpose, a curvature change tracking program was developed to calculate the curvature changes in the main reflector of a VLBI antenna; these changes can directly affect its performance. As the fundamental data for constructing the geometric model, the 3D position coordinates of feature points that are precisely calculated through nontarget-based close-range photogrammetry were used to construct a 3D ellipsoid fitting model to implement the curvature change tracking of the main reflector of the VLBI antenna, which has an intricate geometry. To determine the parameters of the optimal ellipsoid fitting model, the ellipsoid fitting accuracy was enhanced by removing the outliers using the steepest-descent method. Then, to track the curvature changes, the Gaussian and mean curvatures of the fitted ellipsoid model were calculated. The curvature change tracking program can be used to evaluate the structural soundness of the main reflector based on periodic curvature calculations.

Because the objective of this study was to perform a time-series analysis of the curvature calculations of the main reflector to obtain additional evidence for detecting excessive deformations, the accuracy of the ellipsoid fitting was regarded as adequate for tracking purposes. Furthermore, the developed program was demonstrated to have certain economic 
advantages, including lower operational costs and labor, over tracking methods that utilize other types of devices.

\section{Acknowledgments}

This work was supported by the Basic Science Research Program through the National Research Foundation of Korea (NRF) and funded by the Ministry of Education (2016R1A6A3A11931804).

\section{References}

1 P. Sarti, C. Abbondanza, L. Petrov, and M. Negusini: J. Geod. 85 (2011) 1. https://doi.org/10.1007/s00190-0100410-6

2 C. Holst, D. Schunck, A. Nothnagel, R. Haas, L. Wennerbäck, H. Olofsson, R. Hammargren, and H. Kuhlmann: Sensors 17 (2017) 1. https://doi.org/10.3390/s17081833

3 H. G. Maas and U. Hampel: Photogramm. Eng. Remote Sens. 72 (2006) 39. https://doi.org/10.14358/ PERS.72.1.39

4 T. Sahin and M. Unel: J. Math. Imaging Vision 32 (2008) 127. https://doi.org/10.1007/s10851-008-0092-3

5 G. Cross and A. Zisserman: Proc. 1998 IEEE 6th Int. Conf. Computer Vision (ICCV, 1998) 25. https://doi. org/10.1109/ICCV.1998.710697

6 P. Y. Lee and J. B. Moore: Proc. 2004 IEEE 38th Asilomar Conf. Signals, Systems, and Computers (ACSSC, 2004) 131. https://doi.org/10.1109/ACSSC.2004.1399105

7 S. Collings, R. Kozera, and L. Noakes: J. Math. Imaging Vision 34 (2009) 200. https://doi.org/10.1007/s10851009-0142-5.

8 X. Cao and N. Shrikhande: Proc. 1991 IEEE Int. Conf. Systems, Man, and Cybernetics (SMC, 1991) 123. https://doi.org/10.1109/ICSMC.1991.169672

9 A. D. Sappa and M. Rouhani: Proc. 2009 IEEE 16th Int. Conf. Image Processing (ICIP, 2009) 3521. https://doi. org/10.1109/ICIP.2009.5414072

10 E. López-Rubio, K. Thurnhofer-Hemsi, Ó. D. de Cózar-Macías, E. B. Blázquez-Parra, J. Muñoz-Pérez, and I. L. de Guevara-López: J. Math. Imaging Vision 58 (2017) 2. https://doi.org/10.1007/s10851-016-0700-6.

11 P. L. Rosin: Pattern Recognit. Lett. 14 (1993) 189.

12 A. Fitzgibbon, M. Pilu, and R. Fisher: IEEE Trans. Pattern Anal. Mach. Intell. 21 (1999) 476. https://doi. org/10.1109/34.765658

13 M. M. Blane, Z. Lei, H. Çivi, and D. B. Cooper: IEEE Trans. Pattern Anal. Mach. Intell. 22 (2000) 298. https://doi.org/10.1109/34.841760

14 G. Calafiore: IEEE Trans. Syst. Man Cybern. Part A Syst. Humans 32 (2002) 269. https://doi.org/10.1109/ TSMCA.2002.1021114

15 X. Ying, L. Yang, and H. Zha: IEEE Trans. Pattern Anal. Mach. Intell. 34 (2012) 1856. https://doi.org/10.1109/ TPAMI.2012.109

16 W. Gander, G. H. Golub, and R. Strebel: Bit 34 (1994) 558.

17 S. J. Ahn, W. Rauh, and H. J. Warnecke: Pattern Recognit. Lett. 34 (2001) 2283. https://doi.org/10.1016/S00313203(00)00152-7

18 S. J. Ahn, W. Rauh, H. S. Cho, and H. J. Warnecke: IEEE Trans. Pattern Anal. Mach. Intell. 24 (2002) 620.

19 M. Kleinsteuber and K. Hüper: Recent Advances in Optimization and Its Applications in Engineering (Springer, New York, 2010) p. 58.

20 I. Markovsky, A. Kukush, and S. Van Huffel: Numer. Math. 98 (2004) 177. https://doi.org/10.1007/s00211-0040526-9

21 J. J. Lee and M. Shinozuka: Exp. Mech. 46 (2006) 105. https://doi.org/10.1007/s11340-006-3124-2

22 R. Jiang, D. V. Jáuregui, and K. R. White: Measurement 41 (2008) 823. https://doi.org/10.1016/ j.measurement.2007.12.005

23 S. Nishiyama, N. Minakata, T. Kikuchi, and T. Yano: Adv. Eng. Inform. 29 (2015) 851. https://doi.org/10.1016/ j.aei.2015.05.005

24 G. Kortaberria, A. Olarra, A. Tellaeche, and R. Minguez: J. Sens. 2017 (2017) 1. https://doi. org/10.1155/2017/1605943 
25 H. Kim, H. Yun, J. Hwang, and S. Hong: Appl. Sci.-Basel 7 (2017) 1. https://doi.org/ 10.3390/app7111125

26 H. Kim: Noncontact Deformation Monitoring System for Circular-Shaped Complex Structures Using Nontarget Based Close-Range Photogrammetry (Sungkyunkwan University, Seoul, 2018) p. 53.

27 Q. Li and J. Griffiths: Proc. 2004 IEEE Geometric Modeling and Processing (GMP, 2004) 335. https://doi.org/ 10.1109/GMAP.2004.1290055

28 S. Sivapalan, D. Chen, S. Denman, S. Sridharan, and C. Fookes: Proc. 2011 IEEE 8th International Conference on Advanced Video and Signal-Based Surveillance (AVSS, 2011) 355. https://doi.org/10.1109/ AVSS.2011.6027350

29 A. Reza and A. S. Sengupta: Appl. Math. Comput. 314 (2017) 349. https://doi.org/10.1016/j.amc.2017.07.025 